\title{
China como nuevo actor en las inversiones globales
}

\author{
Un análisis de su política inversora y el papel de América Latina
}

China as a New Actor in Global Investments

An analysis of its investment policy and the role of Latin America

Gonzalo Ghiggino $^{1}$

\section{Resumen:}

El expansionismo económico chino ha generado tanto adhesiones como rechazos. En América Latina (AL), la lejanía geográfica y cultural impide el desarrollo de análisis objetivos, desencadenando conclusiones poco fundamentadas.

El presente trabajo reflexionará entorno a las características de las inversiones chinas, tanto a nivel global como en América Latina; por lo tanto, abrodará el papel de la República Popular China (RPCh) en la esfera internacional, la expansión global de las empresas chinas, la política exterior de Beijing hacia esta región, y el patrón inversor de este país en el mundo y en AL.

Palabras clave: China, América Latina, Expansión Económica, Globalización, Inversión, Política Exterior, Estado.

\begin{abstract}
:
The Chinese economic expansionism has generated both accessions and rejections. In Latin America (AL), geographic and cultural distance prevent the development of objective analysis, triggering unsubstantiated conclusions.
\end{abstract}

${ }^{1}$ Candidato a doctor en Estudios Globales, por la Universidad de Shanghái, China. 
This paper will reflect on the characteristics of Chinese investments, both globally and in Latin America; therefore, it will embrace China's role in the international sphere, the global expansion of Chinese companies, Beijing's foreign policy towards this region, and China's investment pattern in the world and in Latin America.

Keywords: China, Latin America, Economic Expansion, Globalization, Investments, Foreign Policy, State.

\section{Introducción}

Dentro de un magno proceso de cambio, el sistema internacional está transitando de un orden unipolar hacia uno multipolar, donde China y otros países emergentes juegan cada vez un papel más preponderante. ${ }^{2}$ Hasta el momento, ningún país -ni Rusia ni China, por citar los casos- puede rivalizar en todos los aspectos con la fuerza internacional de los Estados Unidos.

Si bien, Rusia es cada vez más relevante por su creciente poderío militar, China lo es por su poderío económico. Por un lado, el rol de éste último en el entorno global ha crecido durante los últimos 15 años, conforme a su participación en la economía mundial. Este ascenso ha sido determinado tanto por las condiciones generales como por el activo rol del Estado en la promoción del comercio y de las inversiones.

Por otra parte, la guerra comercial entre la RPCh y EE. UU., desatada en el último año de la mano de la administración de Donald Trump, es una clara muestra del poder de China a nivel internacional. ${ }^{3}$ En este sentido, descifrar el verdadero alcance de su expansión económico/comercial, a través de su política exterior materializada en las inversiones, es una vía válida para entender su influencia y su rol en la economía mundial.

Sin embargo, las lejanías geográficas y culturales atentan contra el desarrollo de análisis profesionales, por lo que el debate en el ámbito académico resulta necesario para

\footnotetext{
2 Zakaria, F. (2008). The Post American World. New York: W.W. Northon and Company.

${ }^{3}$ AFP, R. E. (2018, marzo 23). Trump arremete contra China y le declara la guerra comercial. El País.
} 
entender los matices que la figura de China representa. Por ello, reflexionar acerca del verdadero alcance e influencia de Beijing en América Latina mediante su política exterior y sus inversiones, es una tarea que debe llevarse a cabo para no caer en simplismos. La época contemporánea exige observar sin prejuicios la realidad circundante. En este caso, la presencia de China es un fenómeno incipiente del contexto actual, que genera tanto adhesiones como rechazos.

Una idea fundamental de este objeto de estudio es que la expansión de las empresas y las inversiones chinas han jugado un papel clave en el incremento de su poderío económico. A su vez, el impacto en América Latina es cada vez más evidente. Por ello, el gobierno chino ha diseñado una serie de políticas con el propósito de acercarse la región a nivel político y, a la vez, facilitar y estimular el comercio.

Por tanto, se considera a modo de hipótesis que, si bien el gobierno chino manifiesta políticas de acercamiento hacia $\mathrm{AL}$, las relaciones entre ambas regiones se guían por el patrón de mercado. Por lo que, más allá de las propuestas, el papel de los actores involucrados y las características de estas inversiones se centran en el sector de las materias primas. No obstante, no se puede refutar el esfuerzo chino por mejorar los vínculos y las condiciones comerciales; labor que muchas veces es opacada por el desconocimiento y la desinformación.

Para entender cuáles son estas políticas y cuál es el patrón inversor en el mundo y la región, así como visibilizar los posibles impactos que puedan producirse, se realizará un análisis pormenorizado. En el primer capítulo se expondrá el papel de China en el sistema internacional; en el segundo capítulo se estudiará la expansión de las empresas chinas; en el tercer capítulo se abordará la política exterior de China en América Latina; en tanto que en el cuarto y último capítulo se profundizará acerca del patrón inversor chino y el rol de América Latina.

\section{China en el sistema internacional}

Tras el fin del orden bipolar de la Guerra Fía, una de las principales políticas impulsadas por el gobierno chino fue la promoción del desarrollo, tanto propio como de los demás 
países emergentes. Esta política se fue consolidando paulatinamente. ${ }^{4}$ Hacia finales de los años 90, Beijing se esforzó en adoptar normas de cooperación, particularmente en el área de economía y de seguridad. ${ }^{5}$ En este periodo, la capital defendió activamente a las Naciones Unidas y al Consejo de Seguridad como órganos legítimos de consulta sobre seguridad internacional; también intensificó sus contribuciones a la Asociación de Naciones del Sudeste Asiático (ASEAN), organización a la que propuso un acuerdo de libre comercio. Por otra parte, se unió a la OMC y promovió la cooperación entre países mediante la Organización de Cooperación de Shanghái. ${ }^{6}$

En consecuencia, China emergió como potencia económica durante la primera década del siglo XXI; desde entonces, sus decisiones comenzaron a tener cada vez mayores efectos en la economía mundial. En este marco, su papel en el sistema global adquirió una consideración central, de acuerdo con la preocupación de la comunidad internacional por actualizar las principales insituciones multilaterales, que habían quedado desfasadas en la etapa posterior a la Guerra Fría. ${ }^{7}$

La importancia de la RPCh se manifestó nuevamente en 2015, ante la ralentización del crecimiento de la economía local. Además, diversos factores como la volatilidad del mercado de capitales, las políticas monetarias y el patrón comercial, así como el comportamiento de los inversores impactaron en el crecimiento de su capital. Esta resignificación de su peso financiero y de su impacto internacional fue más pronunciado entre los países de la región Asia-Pacífico.

Cabe destacar que esta zona emergió como nuevo centro de gravitación económica mundial, cuya fortaleza creó nuevas oportunidades para el crecimiento económico chino $\mathrm{y}$, a su vez, el surgimiento de emprendimientos e iniciativas por parte del gobierno.

\footnotetext{
${ }^{4}$ China se comprometió a promover y fortalecer un mundo multipolar donde varios centros de poder trabajen, junto a las Naciones Unidas y otros foros multilaterales, para influir en el curso del desarrollo mundial.

5 Economy and International Governance. (2017). China's Role in the World. Beijing.

6 Shanghai Cooperation Organization Secretariat. (2017, agosto 06). SCO and ASEAN Secretaries-General discuss cooperation prospects. Recuperado el 18 de agosto de 2018, de http://eng.sectsco.org/news/20170806/309746.html

7 Goldstein, A. (2017). "A Rising China's Growing Presence: The Challenges of Global Engagement", in Jacques de L. \& Avery G. (eds.). China's Global Engagement: Cooperation, Competition, and Influence in the 21st Century. Washington, DC: Brookings Institution Press, pp. 1-33.
} 
Respecto a Beijing, los dos proyectos más ambiciosos e importantes promovidos fueron: "La Franja y la Ruta" (OBOR, por su siglas en inglés) ${ }^{8}$ y el Banco Asiático de Inversión en Infraestructura (AIIB, por sus siglas en ingles). El primero fue propuesto en 2013, por el presidente Xi Jinping, y consiste en un un plan de desarrollo de infraestructura y promoción del comercio, a lo largo de una amplia franja que se extiende desde el Sudeste asiático -a través de Asia Central- hasta llegar a Europa. ${ }^{9}$ Con esta iniciativa, China busca liderar el proceso de integración de la economía mundial.

Por otra parte, el AIIB representa un nuevo método de financiación para promover las inversiones en infraestructura que faciliten el comercio. ${ }^{10}$ Significa, tanto para los países asiáticos como para China, la opción de contar con créditos pertinentes con los intereses económicos y comerciales de la región, sin la necesidad de recurrir a otros organismos como el Fondo Monetario Internacional o el Banco Mundial.

Para los países que carecen de infraestructura, la financiación del AIIB representa también la posibilidad de integrarse al circuito comercial impulsado por China. Esto implica que las economías se benefician mediante la incorporación de su sistema productivo y de su comercio exterior, garantizando la entrada al nuevo circuito comercial y, en consecuencia, un crecimiento económico sostenido.

En este marco, las iniciativas representan una oportunidad para evitar los impactos producidos por las tensiones comerciales contemporáneas, fortaleciendo los lazos regionales y expandiendo las rutas comerciales a nivel global. ${ }^{11}$ Para los países emergentes, los planes de integración comercial facilitan la posibilidad tanto de recibir inversiones como de potenciar el mercado. ${ }^{12}$

Sin embargo, estas políticas son criticadas por los detractores del plan del gobierno

\footnotetext{
${ }^{8}$ El nombre más conocido internacionalmente es One Belt, One Road (OBOR), en inglés, en tanto que el nombre en chino es Yi Dai Yi Lu (一带一路).

9 English.gob.cn. (28 de marzo de 2015). Chronology of China's Belt and Road Initiative. Recuperado el 11 de junio de 2018, de The State Council The Peole's Republic of China: http://english.gov.cn/news/top_news/2015/04/20/content_281475092566326.htm

10 Base de datos del Asian Infrastructure Investment Bank, consultada el 12 de junio de 2018 de: https://www.aiib.org/en/about-aiib/index.html

11 Maorong, Zhang. (2016). Significance of Establishing the AIIB. China-United States Exchange Foundation.

12 Elek, A. (2014). The potential role of the Asian Infrastructure Investment Bank. East Asia Forum, Economics, Politics and Public Policy in East Asia and the Pacific.
} 
chino, quienes lo acusan de asumir una actitud expansionista. A su vez, este planteamiento genera ciertas dudas y temores en muchos países emergentes, que temen caer en la trampa de la dependencia económica con Beijing. Al liderar China este proceso de expansión comercial y al ser el principal promotor de la globalización económica, es percibido como un actor de poder que busca incrementar su influencia a nivel mundial. ${ }^{13}$

Estas apreciaciones están basadas en la suposición de que, al igual que los Estados Unidos y los países occidentales, el expansionismo trae como consecuencia la pérdida de soberanía. En este sentido, el desconocimiento de la realidad china afecta las relaciones entre Beijing y los países emergentes. Sobre el expansionismo chino, Robert Kaplan (2010) sostiene que:

China hoy está consolidando sus fronteras terrestres y comienza a girar hacia afuera. Las ambiciones de la política exterior de China son tan agresivas como las de EE. UU. hace un siglo atrás, pero por razones completamente diferentes. China no adopta un enfoque misionero en los asuntos mundiales, buscando expandir una ideología o sistema de gobierno. El progreso moral en las Relaciones Internacionales es un objetivo de los EE. UU., no de China; las acciones del gobierno chino en el exterior son propulsados por su necesidad de asegurarse energía, metales, y minerales estratégicos con el fin de sostener el incremento del estándar del nivel de vida de su inmensa población, que representa aproximadamente $1 / 5$ del total de la población mundial. ${ }^{14}$

La diferencia del enfoque respecto al sistema internacional entre los países occidentales y China, es que los primeros creen en el poder duro y en la intervención mediante la fuerza; en tanto que China busca abrir mercados para fomentar el comercio, sin intervenir de forma directa como poder hegemónico.

El concepto chino de expansionismo está sustentado en un comercio que no está basado en el uso de fuerza, sino que se logra con la firma de acuerdos, así como con la

${ }^{13}$ Chan, Lai-Ha. (2017). "The AIIB and China's Soft Balancing Against the US Pivot to Asia". En Australian Institute of International Affairs (8).

${ }^{14}$ Kaplan, R. (2010). "The geography of Chinese power: how far will China reach on land and the sea?". En Foreign Affairs, No. 89 (3), pp. 22-41. 
cooperación y la promoción de intereses comunes. En este sentido, el experto en política exterior china, Yang Jiemian (2017), afirma que:

Los pensamientos de la corriente principal china están hechos de confucionismo, taoísmo y budismo, todos los cuales abogan por la armonía entre la humanidad y sus entornos sociales y naturales. Estos pensamientos han sido bien cristalizados en la noción de cooperación ganar-ganar para las relaciones exteriores de China. En comparación, los Estados Unidos han desarrollado su pensamiento filosófico en un contexto completamente diferente que se basa en gran medida en la expansión continua de la nación (...) la competencia y la confrontación han constituido el principal tema de interpretación histórica. ${ }^{15}$

Si bien, la política del ganar-ganar -win-win policy- busca seducir a los países emergentes, en especial a los de América Latina, la asimetría comercial y el desconocimiento sobre las políticas chinas generan suspicacias contraproducentes sobre su alcance real y sobre los efectos de las medidas promovidas por el gobierno de Beijing.

Para entender las potencialidades de la expansión de la RPCh en los últimos 20 años, es necesario diferenciarla del proceso de expansión liderado por los Estados Unidos y por Occidente. Si EE. UU. fue el arquitecto de la globalización en los años 80 y 90, a partir de 2008 China fue quien comenzó a asumir ese rol. Tal es la escala de su impacto que, a finales de la segunda década del siglo XXI, está moldeando con mayor presencia la naturaleza de la globalización misma.

En tanto, el comercio y los préstamos internacionales -principalmente en infraestructura, como es el caso de "La Franja y la Ruta" y el AIIB-, son dos de las tres principales características de esta globalización con características chinas. Comercialmente, Beijing es el principal socio de muchos países del mundo ${ }^{16} \mathrm{y}$ actualmente se posiciona como fuente de préstamos a través de sus bancos -el ya mencionado AIIB, el China Development Bank (CDB) y el China Export-Import Bank

\footnotetext{
15 Jiemian, Yang. (2017). "Bringing the Conceptual Gaps: New Imperatives for Managing China-U.S. Relations". En China Quarterly of International Strategic Studies, No. 1(2), pp. 20-21. Shanghai Institutes for International Studies.

${ }^{16}$ Santander Trade Portal. Cifras del Comercio Exterior en China. Recuperado el 5 de julio de 2018, de:https://es.portal.santandertrade.com/analizar-mercados/china/cifras-comercio-exterior
} 
(China ExIm Bank). Por otra parte, hay una tercera característica que define esta globalización, pero con un impacto bastante menor aún; se trata de la reciente internacionalización del Yuan. ${ }^{17}$

Un aspecto a tener en cuenta al analizar este fenómeno liderado por Beijing, es que el desarrollo económico está estrechamente vinculado al proceso de globalización económica. Las reformas del estado que comenzaron a implementarse a fines de los años 70, profundizadas en los 90, fueron posibles gracias al proceso de globalización de la economía mundial simultáneo. La entrada de capitales de inversión -producto de la movilidad global que se expandía hacia los países emergentes-, permitió al gobierno chino impulsar exitosamente sus reformas. ${ }^{18}$

Curiosamente, se ha producido una simbiosis a lo largo de los últimos 40 años, en los que la globalización económica sirvió a China para consolidar su desarrollo y su expansión. Una vez consolidado su proceso de crecimiento económico, pasó a liderar y moldear esta globalización, teniendo un rol cada vez más preponderante.

El éxito chino residió en la capacidad del Estado de re-direccionar las inversiones y el capital entrante al país durante esos años, hacia sectores clave que la economía demandaba. ${ }^{19}$ Si bien, el modelo chino se basa en gran medida en la experiencia de desarrollo de Japón y de Corea del Sur -que combinaron un gobierno altamente activista, una política industrial fuerte y una estrategia dirigida a la exportación-, en la práctica fue y sigue siendo diferente. A fines de los años 90, el gobierno chino se embarcó en la mayor reforma de las State-Owned Enterpirses (SOE's), en medio de una especulación generalizada que supuestamente conduciría a una gran disminución en el tamaño y papel de estas empresas. ${ }^{20}$

Sin embargo, los dirigente reestructuraron y subsidiaron a las SOE's de manera tal

\footnotetext{
17 Jaques, M. (2012). When China Rules the World. The End of the Western World and the Birth of a New Global Order. Penguin Books, Second Edition, p. 596.

18 The World Bank. (16 de julio de 2010). Foreign Direct Investment-the China story. Recuperado de: http://www.worldbank.org/en/news/feature/2010/07/16/foreign-direct-investment-china-story

19 Taube, M. \& O., Mehmet. (2002). "Prospects and Policy Challenges, Foreign Direct Investment in China: Challenges and Prospects for Regional Development". En Main issues on Foreign Investment in China's regional development. OCED.

20 Jaques, M. (2012). When China Rules the World. The End of the Western World and the Birth of a New Global Order. Penguin Books, Second Edition, p. 597.
} 
que, para 2008, todavía poseían el 30\% de los activos totales en el sector industrial y de servicios. Las Top 150 SOE's, lejos de volverse obsoletas, se convirtieron en compañías rentables. El objetivo fue crear un clúster de empresas chinas internacionalmente competitivas, muchas de las cuales eran estatales. Por lo tanto, el corazón del modelo chino es un Estado omnipresente e hiperactivo, que tiene una cercana relación con las SOE's; pero también es una red de conexiones con las mayores firmas del sector privado, consolidándose como la mente maestra de la transformación económica. ${ }^{21}$

La demanda y la expansión de estas empresas fueron canalizadas estratégicamente por el gobierno chino con inversiones hacia el exterior. En el contexto de la globalización económica actual, estas compañías fueron y continúan siendo claves para el crecimiento y la consolidación del papel de Beijing en el escenario internacional. En este proceso, se ubicó a los países emergentes -entre ellos los de América Latina- como fuertes atractivos para su expansión, dada la complementariedad económica existente. ${ }^{22}$

\section{La expansión de las empresas chinas}

De acuerdo con la Comisión Económica Para América Latina y el Caribe (CEPAL), se prevé que cuatro grupos de tecnologías tendrán una influencia particularmente destacada en la economía y la sociedad mundial hacia 2030: las TICs, las tecnologías de automatización y fabricación avanzada, las ligadas a la energía y los recursos naturales, y las de la salud. ${ }^{23}$ En este marco, las empresas chinas ya cuentan con las competencias necesarias para dominar en muchos de los nuevos mercados asociados, por lo que los aspectos sobre competitividad, así como inversión y propiedad intelectual estarán en el centro de los debates comerciales en los próximos años.

El éxito de las compañías para alcanzar este nivel de desarrollo, se debió, en gran medida, a la capacidad de expandirse al resto del mundo. Esto fue posible gracias a la

\footnotetext{
${ }^{21}$ Ibid. pp. 619-620.

${ }^{22}$ Complementariedad en el sentido de que la mayoría de estas empresas son energéticas o mineras y aprovechan la demanda china de recursos naturales y la oferta de los países en vías de desarrollo.

${ }^{23}$ CEPAL. (2014). La Alianza del Pacífico y el MERCOSUR: hacia la convergencia en la diversidad. LC/L 3922. Santiago de Chile, p. 14.
} 
incorporación de China a la Organización Mundial del Comercio (OMC), ya que significó para el gobierno la posibilidad implementar políticas de expansión de las empresas hacia el exterior con más flexibilidad y más libertad.

Para mantener las altas tasas de crecimiento, el gobierno necesitó -y aún necesitaatraer capitales y tecnología; esto no es posible sin una mayor apertura. El protocolo de acceso de entrada a la OMC estipuló, en términos generales, que China debe aumentar la apertura de sus mercados locales para bienes producidos en el exterior, así como para los bienes producidos por empresas extranjeras en el país. ${ }^{24}$ Estas son las llamadas "condiciones de acceso a los mercados". En este sentido, el acuerdo con la OMC ha facilitado la entrada de capital y tecnología, contribuyendo al acelerado crecimiento económico.

Parte de ese capital tecnológico se fue incorporando debido a la expansión de las empresas en el resto del mundo. Esta política de expansión fue denominada como "Going out policy",;25 política que fue promovida por el gobierno central, para impulsar la salida de las empresas locales al resto del mundo. El apoyo estatal resultó clave ya que garantizó que se sostuviera, sin importar la falta de éxito inicial.

Las principales razones de motivación de las empresas por parte del gobierno fueron: 1) entrada a nuevos mercados, 2) acceso a las tecnologías avanzadas, 3) incorporación de expertos en negocios globales, 4) adquisición de marcas reconocidas en el comercio mundial, y 5) posicionamiento como campeones globales. ${ }^{26}$

Los órganos encargados de diseñar y llevar a cabo estas políticas se pueden clasificar en cuatro niveles generales. En el primer nivel está la principal agencia de toma de decisiones, que es el Consejo de Estado -responsable de la gestión general del gobierno y de las decisiones importantes que afectan a la economía y la sociedad.

El segundo nivel está compuesto por las agencias centrales a nivel de ministerio,

\footnotetext{
24 World Trade Organization. Protocols of accession for new members since 1995, including commitments in goods and services. Disponible en: https://www.wto.org/english/thewto_e/acc_e/completeacc_e.htm\#chn

25 China Policy. (2017). China Going Global between ambition and capacity. Beijing.

${ }^{26}$ Backaler, J. (06 de mayo de 2014). 5 Reasons Why Chinese Companies Go Global. En Revista Forbes. $\begin{array}{llllll}\text { Recuperado el de } & 20 & \text { junio de }\end{array}$ https://www.forbes.com/sites/joelbackaler/2014/05/06/5-reasons-why-chinese-companies-go-global/\&refURL= https://www.google.com/\&referrer=https://www.google.com
} 
involucradas en la gestión de inversiones en el extranjero, estas son el Ministerio de Comercio (MOFCOM) y la Administración de Intercambio Estatal (SAFE), así como la Comisión Nacional de Desarrollo y Reforma (NDRC) -que históricamente tuvo responsabilidades claves para aprobar inversiones en el extranjero. ${ }^{27}$

En un tercer nivel se encuentran el Ministerio de Finanzas, el Banco del Pueblo de China, y la Comisión China de Servicios Regulatorios. Estas instituciones han tenido un rol preponderante en el éxito de la expansión de las inversiones y de las empresas chinas.

En el último y cuarto nivel se encuentran los ministerios de línea, como es el caso de los responsables de minería, silvicultura y agricultura. Cada uno está a cargo de las políticas de su propio sector, pero son menos influyentes en la determinación de la política de inversión extranjera correspondiente. ${ }^{28}$ Algunas instituciones como El Consejo Chino para la Promoción del Comercio Internacional (CCPIT) -que depende del MOFCOM-, han jugado un rol de suma importancia, a través de un monitoreo de las iniciativas promovidas por las políticas del Going out. ${ }^{29}$

El rol de instituciones como el CCPIT ha sido fundamental, ya que han promovido el comercio y las inversiones pertinentes con el tipo de industria y su inserción internacional. Esto es importante, ya que se prioriza la promoción de los negocios y de las inversiones en distintas regiones fuera de China, teniendo en cuenta el potencial presente de cada sector económico y cada administración sub nacional; es decir, se promueven las relaciones económicas entre provincias y regiones.

En este contexto, es de destacar el rol de las SOE's provinciales chinas, ya que concentran gran parte de las inversiones en el exterior. Puede observarse también el rol y la presencia estratégica del Estado -ya sea Central como Provincial- en la promoción y el fomento de las inversiones exteriores.

En el caso de las empresas provinciales chinas, los factores que impulsan la Inversión Extranjera Directa (IED) se relacionan más bien con el grado de desarrollo de la

\footnotetext{
${ }^{27}$ Wenbin, Huang \& W., Andreas. (2011). "Analysis of China's overseas investment". En Working Paper

79. Center for International Foresty Research, Bogor, p. 3.

28 Ibíd., p. 4.

${ }^{29}$ Yelery, A. (2014). "China's Going out policy”. En Sub-National Economic Trajectories. Dheli: Institute of Chinese Studies, p. 2.
} 
provincia. Factores como el desarrollo económico provincial, la innovación y la tecnología, así como el ratio de exportaciones el PBI, son determinantes significativos.

Los motivos principales de las IED de las empresas provinciales chinas son dos: la búsqueda de mercado y la eficiencia. ${ }^{30}$ La importancia de esto radica en que las claves para el impulso y el éxito de las relaciones económicas, y sobre todo de las inversiones entre China y América Latina, abren la posibilidad de desarrollar relaciones a nivel regional. Dicho factor facilita el ambiente de negocios entre regiones y provincias similares, acelerando el proceso y asegurando un éxito mayor para las inversiones.

Desde el inicio de su proceso de reforma y apertura, el Estado chino tuvo un rol destacado en el diseño y fomento de estas políticas de desarrollo y expansión mediante sus instituciones. Esta evolución se puede caracterizar a través de cuatro fases de crecimiento en el mercado internacional: la primera fase, a partir de 1978, de internacionalización temprana; la segunda fase, a partir de 1991, de crecimiento en el mercado internacional; la tercera fase, a partir de 2001, de implementación del Going out. Esta última se caracterizó por la presencia global de las empresas chinas, de la que se desprendió una cuarta fase, a partir de 2008, cuando la crisis financiera golpeó a las empresas occidentales y las empresas chinas lograron incrementar su activos intangibles. ${ }^{31}$

A partir del 2001, con la tercera fase, las empresas chinas comenzaron a tener un papel inversor cada vez más importante en América Latina. ${ }^{32}$ La abundancia de materias primas, en particular minerales y fuentes de energías, atrajo el capital de las inversiones chinas, en busca de asegurar el suministro de bienes y productos esenciales para mantener sus altos niveles de crecimiento.

El elevado desarrollo interno, las grandes reservas de divisas y la creciente necesidad de asegurarse recursos naturales, generaron un auge de inversiones en el exterior. A nivel global - excluyendo Hong Kong y otros centros financieros-, los tres primeros destinos de

${ }^{30}$ Chen C. (2013). "Determinants and motives of outward foreign investment from china's provincial firms”. En Trasnational Corporations $\mathrm{N}^{\circ} 23$.

31 Avendaño, L. \& C., Bertha. (2015). "Experiencia de las multinacionales chinas para incursionar en los mercados globales". En Ciencia Administrativa (38-48). Buenos Aires, p. 41.

${ }^{32}$ Dussel Peters, E. \& A., Ariel (coords.). (2015). Beyond Rawl Materials. Who are the Actors in the The Latin America and Caribbean-China Relationship? Buenos Aires: Nueva Sociedad. 
las salidas de inversión extranjera directas (SIED) chinas, fueron los Estados Unidos, Australia y el Reino Unido. Dentro del Top 10 están Francia, Canadá y Alemania. Cabe destacar que, pese al gran salto inversor en la región y más allá de los 106 billones de dólares invertidos por China, aún no hay ningún país de América Latina en este listado. ${ }^{33}$

\section{América Latina en la política exterior china}

América Latina tiene una relevancia estratégica dentro de la política exterior china. El potencial de su mercado, sus recursos naturales y sus ventajas culturales puede impulsar el crecimiento de la RPCh. En este sentido, AL es un escenario de prueba para el despliegue su diplomacia global como potencia. ${ }^{34}$

Desde la perspectiva china, la globalización económica ha sido entendida como un factor positivo para el crecimiento económico. En el ámbito geopolítico, el ascenso de las potencias emergentes generó más espacio para que este país se involucrara con el resto del mundo. Por tanto, desde una perspectiva global, China percibe a América Latina como una accionista con una mentalidad afín (like-minded shareholder) para resolver sus desafíos globales. ${ }^{35}$

Tras la crisis de 2008, iniciada en los países desarrollados, el gobierno y las empresas chinas prestaron más atención a las economías emergentes. China cuenta con recursos financieros, tecnología y capacidad industrial; en tanto que los países emergentes, en especial América Latina, carecen precisamente de capital, infraestructura y capacidad industrial. Indudablemente las ventajas comparativas entre ambas partes son más que complementarias. Al mismo tiempo, los intereses en AL están incrementando. La disminución de la sobrecapacidad productiva y el cambio en el modelo económico chino, donde el crecimiento se basa cada vez más en la innovación y el consumo, generan otras

\footnotetext{
33 Dollar, D. (2017). “China's investment in Latin America”. En Geoeconomics and Global Issues, No.4. Foreign Policy at Brooking, p. 3.

34 Zhang, Wu. (2013). Opportunities along with transformation: A multi-perspective analysis of the China-Latin America relations. Beijing: Economic Management Press, pp. 4-7.

35 Habin, N. (2017). "Las politicas y estrategias de China hacia América Latina y el Caribe”. En Pastrana, E. y U., Gehring (coomps.). La proyección de China en America Latina y el Caribe. Bogotá: Editorial Pontificia Editorial Javierana, p. 102.
} 
demandas. A medida que la necesidad de materias primas y de posicionamiento de sus productos industriales disminuye, surge demanda de productos que requieren otro tipo de inversiones. ${ }^{36}$

No obstante, la demanda de productos agrícolas y mineros por parte de China aún se mantiene en niveles altos; por ello, las inversiones en estos sectores siguen siendo las principales. ${ }^{37} \mathrm{El}$ cambio en el patrón comercial se producirá probablemente a largo plazo, por lo que los países emergentes, en especial de América Latina, deberán aprovechar tanto la demanda actual como la demanda futura. En consecuencia, deberán aplicar políticas públicas y privadas para incorporarse a la nueva tendencia y no perder terreno ante otros competidores.

Actualmente, la estrategia de China hacia AL ha sido pragmática, ponderando la lógica del mercado en vez de la ideología. Lo que busca este país es la cooperación internacional, el respeto y el desarrollo mutuo. ${ }^{38}$ Aunque América Latina no forme parte de OBOR, China le otorga apoyo financiero mediante el establecimiento de numerosos fondos de desarrollo dentro del "Fondo China-CELAC". 39

A su vez, Beijing mantiene vínculos con todas las instituciones multilaterales de la región en diferentes niveles, tales como como la Organización de Estados Americanos (OEA), el Banco Interamericano de Desarrollo (BID), el Mercado Común del Sur (Mercosur) y la Alianza del Pacífico; entre otros. Un importante paso en la dirección de profundizar los lazos entre ambas partes, fue el ingreso de China al BID, que facilitó la inversión en proyectos regionales de infraestructura y el financiamiento de pequeñas empresas.

El gobierno chino ha podido acumular grandes cantidades de reservas internacionales que no pueden ser invertidas o usadas en la economía local; por lo tanto, invertir en otros

\footnotetext{
36 Ibíd. pp. 105-106.

37 Avendaño, R., Menguzio, A. \& M., Sean. (2017). Chinese FDI in Latin America: New Trends with Global Implications. Atlantic Council Adrhien Arsh Latin America Center.

38 Habin, N. (2017). "Las politicas y estrategias de China hacia América Latina y el Caribe”. En Pastrana, E. y U., Gehring (coomps.). La proyección de China en America Latina y el Caribe. Bogotá: Editorial Pontificia Editorial Javierana, p. 109.

39 Departamento de América Latina y el Caribe del Ministerio de Relaciones Exteriores de China. (2016). ABC sobre el Foro China-CELAC.
} 
lugares es la mejor apuesta para fortalecer su presencia internacional y asegurar el futuro del comercio. Estas inversiones no solo fortalecen la imagen de las compañías chinas, sino que pueden contribuir en la consolidación un perfil exportador diversificado en AL.

Asia yAmérica Latina son las dos regiones con mayor concentración de países con mercados emergentes. Durante la primera década del siglo XXI, se ha presenciado el ascenso asiático con el traspaso del eje de la economía mundial del Atlántico hacia el Pacífico. De acuerdo con CEPAL, en el 2011 el crecimiento del PBI mundial fue del 4,4\%; los países industrializados contribuyeron al 1,22\% y los países y regiones en desarrollo aportaron el 3,18\%. ${ }^{40}$ La contribución de los países en desarrollo significó el $72 \%$ del crecimiento económico, lo cual refleja que en la era post-crisis ambas regiones se han convertido en el principal motor del crecimiento económico mundial. ${ }^{41}$

En este marco, el gobierno chino promueve las relaciones con América Latina, consciente de la importancia del comercio y de las inversiones compartidas. La política china hacia esta región se basa en el respeto común y en la búsqueda del beneficio mutuo, bajo los preceptos del Win-win.

En el año 2014, en la "Cumbre sino-latinoamericana" celebrada en Brasilia, el presidente Xi Jinping pronunció el discurso "Para lograr un desarrollo colaborativo hacia un destino común". ${ }^{42}$ Con este mensaje propuso la meta de construir una relación integral de cooperación entre las regiones, con el fin beneficiar mutuamente su desarrollo económico. Para lograr este fin, el líder sugirió varias ideas:

a)Elevar la cooperación integral sino-latinoamericana a un nivel más alto.

b)Fomentar una relación bilateral caracterizada por una nueva estructura colaborativa llamada "Cinco en uno".

c)Construir un marco nuevo de cooperación entre las dos regiones bajo el modelo " $1+3+6 "$.

\footnotetext{
${ }^{40}$ CEPAL. (2011). People's Republic of China and Latin America and the Caribbean: Ushering in a new era in the economic and trade relationship, p. 11.

${ }^{41}$ Ibid., p. 12.

42 Observatorio de la Política China. (2014). Entrevista del presidente Xi Jinping con medios de comunicación de Brasil, Argentina, Venezuela y Cuba. Recuperado de: http://politica-china.org/areas/politica-exterior/entrevista-por-escrito-del-presidente-xi-jinping-con-mediosde-comunicacion-de-brasil-argentina-venezuela-y-cuba
} 
d)Impulsar la cooperación bilateral por medio del "Foro sino-latinoamericano".

e)Promover las relaciones colaborativas Sur-Sur por medio del fortalecimiento de los vínculos entre China y América Latina.

f)Crear una red de cooperación caracterizada por la integralidad y la comprensión.

g)Tener como objetivo la realización del "sueño latinoamericano".

h)Luchar a la par para llegar a un destino común: la modernización total de los países. ${ }^{43}$ Estas propuestas tienen como objetivo la profundización del desarrollo colaborativo entre las dos regiones; también son una base para futuros diálogos más detallados. Ambas partes tienen un punto de interés común: el fortalecimiento de cooperaciones en más aspectos y sectores de la economía, a través de un desarrollo compartido.

Hay que destacar la propuesta de sugerencias prácticas, concretas y factibles, que integran el nuevo marco cooperativo " $1+3+6$ ". Dentro de este esquema, el número " 1 " se refiere a localizar los problemas que enfrentan las dos regiones para establecer los objetivos y las perspectivas de la cooperación sino-latinoamericana, y luego crear una dinámica basada en la integración de las estrategias económicas de ambas partes.

En tanto, el número "3" hace referencia a los tres motores fundamentales para el desarrollo de esta cooperación en su máximo potencial: el comercio, la inversión y el financiamiento. Por último, el número " 6 " representa los seis sectores cruciales dentro de este proceso: la energía, la infraestructura, la agricultura, la manufactura, la innovación científica y tecnológica, y las tecnologías informáticas. Todas estas industrias son de mayor importancia tanto para el acoplamiento sectorial entre las dos regiones, como para la profundización y el fortalecimiento de la cooperación económica.

Además de los puntos mencionados, el presidente $\mathrm{Xi}$ también propuso planes concretos para llevar a cabo en el futuro. Estas iniciativas tienen como objetivo expandir la escala del comercio entre China y los países latinoamericanos a 500 billones de dólares, en un periodo de diez años, y realizar una inversión total de 250 billones en la región durante el mismo plazo. ${ }^{44}$

\footnotetext{
${ }^{43}$ Instituto de Mercados Emergentes Universidad Normal de Beijing. (2015). El nuevo marco de la cooperación económica sino-latinoamericana. Para un desarrollo común, práctico y mutuamente beneficioso.

${ }^{44}$ Ministerio de Comercio de China. (2014). Comunicado sobre la inversión directa China en el extranjero, 2013. China: China Statistics Press.
} 
Por otra parte, se mencionó la creación de un fondo exclusivo de 10 billones de dólares para la construcción de infraestructura en AL, monto que luego se incrementó a 20 billones; el ofrecimiento de préstamos preferenciales de 10 billones de dólares de China hacia los miembros de la CELAC; el lanzamiento formal de un fondo exclusivo para la colaboración sino-latinoamericana, con una promesa de 5 billones de dólares, y el establecimiento de un fondo de 50 millones de dólares para la cooperación agrícola entre las dos partes. ${ }^{45}$

Con estos anuncios, se aprecia el interés de China por mejorar los vínculos con América Latina, ponderando el incremento de las inversiones y el desarrollo comercial cualitativo. En este marco, la RPCh traza una política exterior concreta, donde tanto América Latina como los países emergentes son igual de prioritarios que los países desarrollados.

\section{El patrón inversor chino y el rol de América Latina}

Si bien, la mayoría de las inversiones chinas se orientan a los países desarrollados -más estables en cuanto a seguridad jurídica-, en ciertos casos hay exepciones notables. Estas inversiones se guían principalmente por la lógica de mercado, y el hecho de que se apliquen a países poco estables se debe a que muchas de ellas son realizadas por compañías estatales; por lo tanto, estas empresas no tienen la misma presión que las compañías privadas de asegurar un alto margen de retorno.

Los líderes chinos quieren que las grandes empresas estatales se conviertan en "campeonas globales". En esta trayectoria, hay ciertos aprendizajes involucrados, y por añadidura, algunas pérdidas. A pesar de los riesgos, estas compañías estatales no son tan susceptibles a las pérdidas, dado que no tienen grandes restricciones presupuestarias.

En este contexto, la estrategia Going out tiene diversas aristas; una de ellas es la ayuda diplomática y financiera del gobierno; que se concreta por lo general en préstamos con bajos intereses a empresas o en soporte financiero para otros Estados. ${ }^{46}$

\footnotetext{
45 Instituto de Mercados Emergentes Universidad Normal de Beijing. (2015). El nuevo marco de la cooperación económica sino-latinoamericana. Para un desarrollo común, práctico y mutuamente beneficioso, p. 8.

46 The Economist. China's "going out" strategy. Recuperado el 5 de septiembre de 2018, de:
} 
A partir de 2004, el gobierno y los bancos estatales comenzaron a ofrecer acceso preferencial a financiación, beneficios tributarios y demás incentivos con el fin de facilitar la salida de las empresas al mercado internacional. Este entramado de préstamos y financiamiento consolidó a China como el segundo acreedor global en el año 2015, con una inversión exterior de 2,4 trillones de dólares. ${ }^{47}$

La mayoría de las inversiones chinas en el exterior, en especial en América Latina, tuvieron un crecimiento exponencial a partir de 2009. Si bien, con la crisis financiera mundial las inversiones de los países desarrollados se desplomaron, la tendencia china fue de continuo crecimiento. Esto fue posible gracias al fuerte apoyo estatal brindado durante esos años. A diferencia de las demás empresas inversoras de los países desarrollados, las empresas chinas no afrontaron una contracción del crédito y pudieron aprovechar las oportunidades en el exterior con menos competencia extranjera. ${ }^{48}$

Es necesario ponderar que el comportamiento inversor de estas empresas se muestra altamente influenciado por el tipo de políticas gubernamentales aplicadas. Gracias a los mecanismos de control, el gobierno puede distribuir la salida de inversiones extranjeras directa de acuerdo con los objetivos e intereses de la economía estatal.

Pero las IED chinas y el proceso de internacionalización de sus empresas no han transitado siempre por el mismo camino. Por una parte, las empresas chinas han buscado expandirse con mayor interés en los países desarrollados, debido a las condiciones óptimas que presentan estas naciones. En cambio, el mercado latinoamericano no posee las características que demandan estas empresas.

Por otra parte, las inversiones de las empresas chinas en la región pueden focalizarse en el mercado; pero la asociación con empresas locales para desarrollar el mercado es más bien una tendencia y un objetivo a largo plazo. En este sentido, las inversiones en América Latina, son realizadas principalmente por las empresas energéticas, mineras y del sector financiero. Por tanto, las transacciones chinas en los países desarrollados se han

https://www.economist.com/free-exchange/2009/07/21/chinas-going-out-strategy

${ }^{47}$ Dollar, D. (2016). "China as a global investor". En China's new Sources of Economic Growth Vol. 1. ANU Press, pp. 197-213.

48 De Vuele, F. \& V., David. (2010). The global crisis, Foreign Direct Investment and China: Developments and Implications. Brussels Institute of Contemporary China Studies, p. 7. 
centrado en la compra o fusión de compañías locales y en la adquisición de tecnologías; mientras que en los países en vías de desarrollo se han realizado en materias primas. ${ }^{49}$

Ésta última no constituye un monopolio de los países en vías de desarrollo. Algunos países desarrollados como Estados Unidos, Canadá y Australia -que cuentan con grandes reservas de recursos estratégicos para China-, han recibido inversiones millonarias en este rubro. El problema para los países de América Latina es que las inversiones chinas en materias primas representan más del $70 \%$ del total. ${ }^{50}$

Las IED chinas en el exterior obedecen a la lógica del mercado, pero se orientan profundamente a los intereses nacionales. Pero el carácter macroeconómico de los países receptores constituye un importante condicionante. En este sentido, los más puntos más importantes son:

-El deseo de las empresas multinacionales chinas de ampliar su mercado.

-El tamaño del PBI es un determinante significativo para la atracción de las IED chinas.

-Una moneda débil en el país receptor es más favorable para los proyectos de inversión de las multinacionales, por lo que el tipo de cambio es un impulsor clave.

-Una inflación estable y predecible es un determinante favorable para las IED chinas, ya que genera mayor confianza y certidumbre para la planificación a largo plazo.

-Un mayor grado de apertura económica de la economía receptora es más atractivo para las IED chinas.

Finalmente, el factor más relevante de todos es la dotación de recursos naturales, ya que China busca mediante este tipo de inversiones asegurar el suministro continuo de materias primas, necesario para el desarrollo de su economía. ${ }^{51}$

Dada la posición estratégica que representan, las empresas mineras han sido una de las más beneficiadas del Going out, ya que la búsqueda de recursos naturales es una política de estado caracterísitica del gobierno chino.

\footnotetext{
49 The Economist Intelligence Unit. (2017). China Going Global Investment Report.

50 American Enterprise Institute. (s.f.) China Global Investment Tracker. Obtenido de: http://www.aei.org/china-global-investment-tracker/

51 Zhang, Xiaoxi \& D., Kevin. (2011). "The determinants of China's outward foreign direct investment". En Emerging Market Review No. 4(12), pp. 389-398.
} 
La ambición del Estado de transformar las empresas en grandes campeonas globales y garantizar los recursos para su desarrollo, es una realidad. Primero, porque las empresas, principalmente las mineras, se ubican entre las más grandes en cuanto al mercado y la capitalización a nivel global; a su vez, China cuenta con la mayor cantidad de empresas mineras en todo el mundo. ${ }^{52}$ Segundo, porque China, gracias a las inversiones realizadas por estas empresas, ha logrado entrar en mercados con abundantes materias primas, como Australia, Canadá, América Latina y África.

La expansión y el éxito comercial de las empresas chinas ha sido fenomenal y aún tienen un largo trayecto por recorrer en la incorporación de nuevos mercados. La competitividad que presentan, su efectividad y el permanente apoyo estatal a sus políticas de expansión, las hacen prometedoras en cuanto a capacidad de inversión y sostenimiento a lo largo del tiempo. Ante la poca estabilidad y capacidad de retorno que ofrecen muchos países, las empresas chinas son las únicas que puede afrontar este desafío.

De cara al futuro, estas empresas serán capaces de incrementar su participación en el Market share de las inversiones y el comercio, principalmente de las mineras. En este sentido, el desafío para los países emergentes -específicamente para América Latina-se presenta no solo en la atracción de las inversiones, sino también en brindar un marco adecuado y saber diversificar los intereses chinos en la región.

China se posicionó como uno de los principales impulsores de las fusiones y adquisiciones, al tiempo que se consolidó como el segundo inversor mundial, detrás de EE. UU. ${ }^{53}$ En tanto, la tendencia de las inversiones chinas en el mundo muestra que las IED se dividen en regiones; a su vez, en cada región es distinta la inversión realizada.

\footnotetext{
${ }^{52}$ Las empresas Jianxi Cooper Corporation Limited (JCCL), China Shenghua Energy Company Limited (CSEC), y Zijing Mining Grouop están entre las compañías mineras más grandes del mundo. Solo China ostenta este record. (Ver: Jammula, A. K. (21 de junio de 2018). The world's biggest mining companies in 2018. Mining Technology).

${ }^{53}$ Casi el 60\% del valor de las fusiones y adquisiciones realizadas por empresas chinas entre 2015 y 2016 se concentró en Europa y Estados Unidos. Asia sigue siendo un destino importante para las empresas chinas, que representan el $23 \%$ de las adquisiciones totales en esa región. Mientras tanto, un pequeño porcentaje de las adquisiciones de empresas chinas, apenas el 4\% del total, se realizó en América Latina y el Caribe. (Ver: Investment and the Digital Economy. (2017) World Investment Report. Geneva: Economic Commission for Latin America and the Caribbean, on the basis of official figures and United Nations Conference on Trade and Development).
} 
Por ejemplo, en el año, 2017 Europa fue el principal destino de las IED, con un monto de 258 billones de dólares -siendo el rubro "Agriculture and Foresty" el más importante, al concentrar el 35\% de las inversiones. En este año incidió la compra de la compañía Suiza Syngenta por parte de la empresa ChemChina, por lo que las inversiones chinas en el sector agrícola en Europa representaron el 45\% del total; el máximo en ese rubro en los últimos 15 años. ${ }^{54 \_55}$ El segundo sector fue el de "Entretainment and Travel" y el tercero el de "High Tech and Transport". 56

Por otra parte, el continente asiático -incluida Rusia-, fue el segundo destino de las IED por más de 200 billones, siendo el rubro "High Tech and Transport" el más importante, seguido muy de cerca por "Mining, Chemical and Energy". EE. UU. ocupó el tercer lugar, con una inversión de 169 billones, de los cuales cerca del 40\% se destinaron a los rubros "High Tech and Transport". América Latina ocupó la cuarta posición, con un monto de 143 billones, destinándose más del 70\% al rubro "Mining, Chemical and Energy". 57

África y Oceanía -principalmente Australia- ocuparon el quinto y sexto lugar respectivamente. En el caso de África, más del 80\% de los 106 billones que representaron las IED se centraron en el rubro "Mining, Chemical and Energy"; mientras que en Oceanía, más del 40\% de los 90 billones se concentraron en el mismo aspecto, en tanto que el rubro "Hig Tech and Transport" representó más del 30\% del total. ${ }^{58}$

En su reciente publicación, Exploring new forms of cooperation between China and Latin America and the Caribean (2018), CEPAL afirma que cerca de 90 mil millones de dólares ingresaron a América Latina y el Caribe desde China entre 2005 y 2016, lo que

\footnotetext{
${ }^{54}$ Analytical Credit Rating Agency. China capital outflow restrictions give way to new opportunities for CIS countries. Recuperado el 16 de julio de 2018, de: https://www.acra-ratings.com/research/344

55 La compra de Syngenta significó un importante paso en la consolidación de las multinacionales chinas en el rubro de Agricultura.

${ }^{56}$ Ministry of Commerce, Public Services. Investment project information data base. Recuperado el 8 de agosto de 2018, de: http://project.mofcom.gov.cn/1800000091_10000108_8.html

57 The Statistics Portal. (s.f.). Breakdown of China's outward foreign direct investment flows in 2015, by industry (in million U.S. dollars). Recuperado de: https://www.statista.com/statistics/722150/china-outward-fdi-flows-by-industry/

${ }^{58}$ Huanga, B. \& Xia, Le. (2018). "China ODI from the Middle Kingdom: What's the next after the big turnaround?". En BBVA Executive Summary, China Economic Watch, February.
} 
representa aproximadamente el 5\% de las entradas de IED. ${ }^{59}$ Sin embargo, es muy probable que haya un marcado aumento tanto en el valor absoluto como en la participación de IED en la región.

Se estima que en 2017, la inversiones chinas en América Latina y el Caribe fueron de más de 25 mil millones, lo que equivale a alrededor del 15\% del total. Brasil recibió el 55\% de las inversiones realizadas en la región desde 2005, incluidas las estimaciones de 2017, seguido por Perú, con un 17\%, y Argentina, con el 9\%. Por tanto, los tres principales países receptores representan el $81 \%$ de las entradas. ${ }^{60}$

Por otra parte, la tendencia muestra que a partir del 2013 la mayoría de las IED se realizaron a través de la Iniciativa de "La Franja y la Ruta". Esta inversión presenta un crecimiento sostenido durante los últimos cuatro años, abarcando países de Asia y Europa. La mayor cantidad de estas inversiones se destinaron a proyectos de infraestructura para promover y facilitar el comercio entre las dos regiones.

Según datos del Ministerio de Comercio de la RPCh, la mayoría de las inversiones se realizaron en los países a través de OBOR. En 2017, las inversiones en 59 países a lo largo de la iniciativa alcanzaron los 14,36 billones de dólares. El valor total de los proyectos contractuales recientemente firmados en 61 países ascendió a 144,32 mil millones de dólares, ocupando el 54,4\% del valor total de lo invertido en el extranjero. ${ }^{61}$

Las inversiones a través de la iniciativa representan el plan más ambicioso de obras en el exterior, lo cual tiene un gran impacto en la distribución de las IED a nivel internacional. La mayoría de las inversiones en infraestructura se realizan a través de OBOR, sobre todo en los países asiáticos y europeos.

Esta realidad revela la importancia de ser parte de "La Franja y la Ruta". Si bien, la tendencia de las IED chinas en la región es la del continuo crecimiento, la lenta diversificación y la falta de estrategias claras por parte de los gobiernos latinoamericanos son problemas que se deben encarar ante la necesidad de obras de infraestructura en la región. La

59 CEPAL. (2018). Exploring new forms of cooperation between China and Latin America and the Caribbean. Second Ministerial Meeting of the "Forum of China and the Community of Latin America and the Caribbean States". Santiago de Chile, p. 56.

60 Ibid.

61 Ministry of Commerce, Public Services. Investment project information data base. Recuperado el 8 de agosto de 2018, de: http://project.mofcom.gov.cn/1800000091_10000108_8.html 
clave radica en el papel del Estado y en cómo la región diseña y afronta políticas concretas para aprovechar las oportunidades que ofrecen las inversiones chinas.

\section{Conclusiones}

Como se ha analizado, las relaciones entre China y AL involucran complejas condiciones basadas en la división del trabajo. ${ }^{62}$ Los intereses en la región, representados a través de sus empresas y de sus inversiones, se basan principalmente en la explotación de los recursos naturales, mayormente los minerales.

Si bien, las grandes empresas estatales lideran el proceso de inversión en el extranjero, se espera que poco a poco más compañías privadas chinas incrementen su participación. La participación de china en AL, como parte de la política Going out, debe entenderse dentro de un contexto más amplio de globalización para China y el resto del mundo; cuyos términos cualitativos y cuantitativos- varían según la región.

Dado que las inversiones chinas se basan en la lógica de mercado -guiada, a su vez, por los intereses nacionales-, deben ser entendidas en el marco de las políticas estado-céntricas, donde el diseño de políticas de expansión dictadas por el gobierno central de Beijing son fundamentales. Este patrón indica que la rentabilidad, de acuerdo con los intereses chinos, determinará las inversiones en un determinado país.

En este marco, la planificación estatal y la orientación de las inversiones juegan un papel clave. Por ello, no solo es necesario direccionar las inversiones, sino que se requiere de una política exterior diseñada para menguar su impacto negativo, mediante la búsqueda de políticas de acercamiento y de consenso. En este sentido, China plantea acercarse a América Latina, donde el multilateralismo juega un papel de suma importancia.

Es claro que la RPCh manifiesta un intento por mejorar las condiciones en las relaciones económicas con AL. Políticas de acercamiento, así como proyectos de infraestructura inclusivos -dentro y fuera de América Latina-, son la tentativa más clara de esta intención. Por tanto, iniciativas como "La Franja y la Ruta" se consolidan como estrategias para el desarrollo y resultan fundamentales para mejorar el nivel e impacto de las inversiones.

${ }^{62}$ Armony, A. \& S., Julia. (2012). "From Going Out (zou quchu) to Arriving In (desembarco): Constructing a New Field of Inquiry China-Latin America Interactions". En The China Quarterly No 209. Cambridge University Press on Behalf of School of Oriental and African Studies, pp. 1-17. 
orientando | Temas de Asia Oriental, Sociedad, Cultura y Economía

Sin duda, la lejanía geográfica y cultural, añadida a las experiencias propias de América Latina, atenta contra la posibilidad de encarar las relaciones de una manera pragmática. Además, la falta de una clara política estatal por parte de las naciones implicadas dificulta que sus dirigentes puedan visualizar los resultados en el mediano plazo. La discusión entonces está abierta, y es importante entender el contexto internacional, así como los alcances y los objetivos de la política exterior por parte de China y el impacto de sus inversiones. 